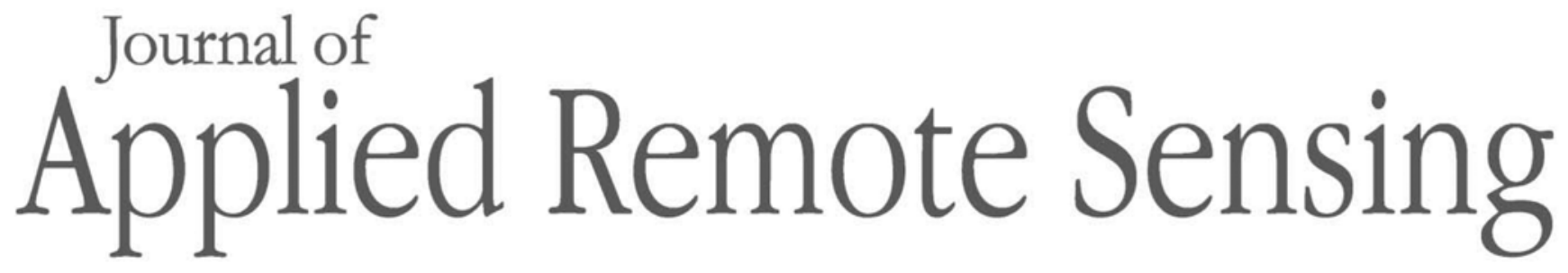

RemoteSensing.SPIEDigitalLibrary.org

\title{
Simulations and experimental results of cloud thermodynamic phase classification with three SWIR spectral bands
}

\author{
Martin Jan Tauc \\ David W. Riesland \\ Laura M. Eshelman \\ Wataru Nakagawa \\ Joseph A. Shaw
}

Martin Jan Tauc, David W. Riesland, Laura M. Eshelman, Wataru Nakagawa, Joseph A. Shaw,

"Simulations and experimental results of cloud thermodynamic phase classification with three

SPE SWIR spectral bands," J. Appl. Remote Sens. 13(3), 034526 (2019), 


\title{
Simulations and experimental results of cloud thermodynamic phase classification with three SWIR spectral bands
}

\author{
Martin Jan Tauc, ${ }^{a}$ David W. Riesland, ${ }^{\text {a,b }}$ Laura M. Eshelman, ${ }^{\text {a,c }}$ \\ Wataru Nakagawa, ${ }^{a}$ and Joseph A. Shaw ${ }^{\mathrm{a}, *}$ \\ ${ }^{a}$ Montana State University, Department of Electrical and Computer Engineering, \\ Bozeman, Montana, United States \\ ${ }^{b}$ U.S. Army Futures Command, Combat Capability Development Center, Aviation and \\ Missile Center, Redstone Arsenal, Alabama, United States \\ ${ }^{\mathrm{c}}$ Polaris Sensor Technologies Inc., Huntsville, Alabama, United States
}

\begin{abstract}
Knowing the thermodynamic phase of a cloud-whether it is composed of spherical water droplets or polyhedral ice crystals-is critical in remote sensing applications and in climate studies. Liquid water and ice have different absorptive properties in certain spectral bands that can be exploited to identify the phase of clouds using ground-based, passive remote sensing. Our simulations found that ground-based radiance measurements at three spectral channels $(1.55,1.64$, and $1.70 \mu \mathrm{m})$ provide improved discrimination when analyzed in three spectral dimensions as opposed to previous approaches based in two-dimensional parameter space. Our simulations show that these bands provide good discrimination between liquid-water and ice clouds when the optical depth is large. We also show measurements from a ground-based spectrometer confirming the cloud-phase sensing ability of these three channels, with validation provided by a dual-polarization lidar system. () The Authors. Published by SPIE under a Creative Commons Attribution 4.0 Unported License. Distribution or reproduction of this work in whole or in part requires full attribution of the original publication, including its DOI. [DOI: 10.1117/1.JRS.13.034526]
\end{abstract}

Keywords: cloud thermodynamic phase; atmospheric radiation; remote sensing.

Paper 190270 received Apr. 9, 2019; accepted for publication Aug. 28, 2019; published online Sep. 23, 2019.

\section{Introduction}

The shape, size, orientation, concentration, and thermodynamic phase of clouds must be known to understand and predict the way clouds influence Earth's climate ${ }^{1-12}$ and affect Earth-space communication. ${ }^{13-15}$ The intricacies of how clouds interact with each other and with aerosols can become very complex, but their basic role is to either cool or heat the planet. The cloud thermodynamic phase (CTP) is not only an important factor in cloud development and evolution, but it is also a prerequisite to inferring other cloud properties, such as particle size and shape. Broadly speaking, water clouds, which are composed of spherical droplets, tend to deflect warming rays from the sun; whereas ice clouds, which can be composed of various crystal structures,$^{16}$ tend to heat the earth, and thus CTP is important in weather and climate models. ${ }^{6,17,18}$ Furthermore, remote sensing systems cannot effectively determine particle shape, size, and other properties until the thermodynamic phase is known., $3,10,11,19$

Although in situ measurements of CTP are the most accurate because they physically measure the liquid water content, they are also inconvenient since they require an aircraft to fly through clouds. Remote sensing systems, on the other hand, can be deployed on the ground or on satellites, thereby providing frequent measurements. Of the available active sensor systems, dual-polarization lidar discriminates between liquid-water and ice clouds most directly and likely with the greatest accuracy. ${ }^{7,20-22}$ However, lidar systems require scanning to monitor a large segment of the atmosphere and consume more power than passive sensing systems.

*Address all correspondence to Joseph A. Shaw, E-mail: joseph.shaw@montana.edu 


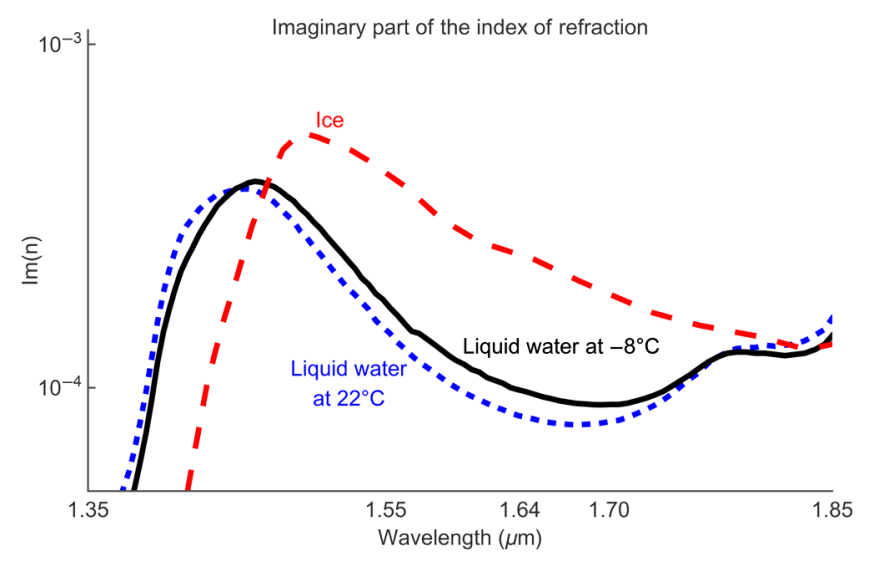

Fig. 1 The different values for the imaginary part of the refractive index between liquid water and ice can be exploited to classify cloud thermodynamic phase. Even supercooled water (black solid line) shows very similar properties to room-temperature water, ${ }^{26-28}$ which is important because supercooled-liquid water clouds are common. ${ }^{9}$

In many cases, such as spectrometers ${ }^{23}$ and spectral radiometers, ${ }^{11}$ passive systems are less expensive, provide a larger field of view without scanning, and are thus easier to deploy and run continuously than active systems. ${ }^{7,20,22}$ For example, cloud lidars typically have a field of view in the microradian to milliradian range, and nearly any passive system exceeds this by at least an order of magnitude. Analogous to the NASA AERONET program that deploys solar radiometers at locations around the globe,$^{24}$ a similar network of passive sensors could be deployed to detect CTP.

A great amount of work has gone into classifying CTP with infrared spectral bands and can be traced back to $1960 .^{25}$ The key to nearly all studies using spectral bands to determine CTP is the difference in the imaginary part of the index of refraction (and thus the absorption) between ice and liquid water. ${ }^{26-28}$ The difference between the imaginary part of the refractive indices in the band of 1.35 to $1.85 \mu \mathrm{m}$ can be seen in Fig. 1 for the spectral range used in our study, although others have exploited the difference between the two indices in other regions of the spectrum. In the remainder of this section, we briefly mention a number of passive methods developed for CTP classification with various advantages and disadvantages (to the best of our knowledge, there is no generally agreed upon accuracy requirement for CTP classification).

Since sunlight absorbs differently in liquid water than in ice, the thermodynamic phase of a cloud can be interpreted from ratios of scattered solar radiance measured in at least two spectral bands. Table 1 summarizes some key contributions to CTP classification with radiance ratios. Pilewskie and Twomey ${ }^{23}$ were the first to use this method by measuring the vertical profile of clouds while the sun was at a zenith angle of $45 \mathrm{deg}$. The spectra of ice and liquid-water clouds differed greatly in their band of interest, but they did not have independent validation of the cloud phase. Baum et al. ${ }^{16,29}$ simulated moderate-resolution imaging spectroradiometer (MODIS) data by flying an aircraft above clouds in a campaign called the subsonic aircraft contrail and cloud effects special study. They used visible and shortwave infrared (SWIR) channels at 0.65, 1.63, and $1.90 \mu \mathrm{m}$ to enhance their thermal infrared-based process of deducing CTP. They reported problems with multilayer clouds and clouds with optical depths of $<2$.

Knap et al. ${ }^{5}$ focused on the slope of absorption as a function of wavelength to infer CTP. Their work capitalized on the fact that at $1.67 \mu \mathrm{m}$, the slope of the liquid water absorption spectrum is essentially zero, whereas the slope of the ice spectrum is negative. They defined the slope as the normalized difference between the 1.70- and $1.64-\mu \mathrm{m}$ bands. They simulated data from aircraft flying over land, water, or snow with clouds in between. Their experimental measurements were done from an aircraft over the ocean with a nadir-pointing instrument. They noted that the greatest challenges in these methods were detecting CTP over certain surfaces, such as snow and rock, especially when the optical depth was low, and in differentiating between ice and mixed-phase clouds. 
Table 1 A table of work in the SWIR bands for CTP classification shows the large effort that has gone into determining the thermodynamic phase of a cloud with spectral bands.

\begin{tabular}{|c|c|c|}
\hline Authors & Year & Method \\
\hline Pilewskie and Twomey ${ }^{23}$ & 1987 & 1.55 to $1.75 \mu \mathrm{m}$ \\
\hline Baum et al. ${ }^{16,29}$ & 2000 & $0.65,1.63$, and $1.90 \mu \mathrm{m}$ \\
\hline Knap et al. ${ }^{5}$ & 2002 & 1.64 and $1.70 \mu \mathrm{m}$ \\
\hline Acarreta et al. ${ }^{19}$ & 2004 & 1.55 to $1.67 \mu \mathrm{m}$ \\
\hline Chylek and Borel ${ }^{30}$ & 2004 & 0.87 and $1.60 \mu \mathrm{m}$ \\
\hline King et al. ${ }^{31}$ & 2004 & $0.66,1.62$, and $2.13 \mu \mathrm{m}$ \\
\hline Chylek et al. ${ }^{32}$ & 2006 & 0.66 and $2.13 \mu \mathrm{m}$ \\
\hline Ehrlich et al. ${ }^{33}$ & 2008 & 0.86 to $1.70 \mu \mathrm{m}$ \\
\hline Martins et al. ${ }^{8}$ & 2011 & 2.10 and $2.25 \mu \mathrm{m}$ \\
\hline Jäkel et al. ${ }^{11}$ & 2013 & 1.55 and $1.70 \mu \mathrm{m}$ \\
\hline Thompson et al. ${ }^{10}$ & 2016 & 1.4 to $1.8 \mu \mathrm{m}$ \\
\hline
\end{tabular}

Acarreta et al. ${ }^{19}$ built on the work of Knap et al. and found that the addition of spectral fitting in the range of 1.55 to $1.67 \mu \mathrm{m}$ increased the robustness of the classification method. Their findings from satellite spectra also suggested that the ground reflectance can be problematic when trying to classify CTP from space, and thus a good understanding of the ground reflectance is very helpful but not always available. Furthermore, they ignored cloud optical depths below 3 and found that the solar zenith and viewing angle only had a small effect on CTP classification.

A similar instrument to MODIS is the U.S. Multispectral Thermal Imager (MTI), which was used by Chylek and Borel $^{30}$ to determine CTP. Their simulations, using the MODTRAN radiative transfer code and data from the MTI, identified the reflectance ratio between 0.87 - and $1.6-\mu \mathrm{m}$ spectral bands as a good tool for classification. In a later study, Chylek et al. ${ }^{32}$ used 0.66- and $2.13-\mu \mathrm{m}$ bands to classify CTP. They used thermal infrared bands to identify the temperature of the cloud and noted that signatures above $0^{\circ} \mathrm{C}$ were liquid-water clouds, ice clouds were below $-40^{\circ} \mathrm{C}$, and mixed phase clouds could exist in between. They reiterated the difficulties of the ground reflectance interfering with CTP classifications for certain ground types.

Ehrlich et $a .^{33}$ used data from high-altitude aircraft to develop three separate methods for CTP identification. Their first method relied on the radiance difference in spectral bands at 1.55 and $1.70 \mu \mathrm{m}$, normalized by the radiance at $1.64 \mu \mathrm{m}$. This method was validated mostly with in situ instruments. The second method used principal component analysis on simulated ice clouds near the $1.55-\mu \mathrm{m}$ band and water clouds in the $1.60-$ to $1.70-\mu \mathrm{m}$ band, and normalized them to the $0.86-\mu \mathrm{m}$ band. Their first two methods, much like the methods from other researchers, did not distinguish CTP for clouds with low optical depth (below 2). Their third method relied on the different directional scattering that occurs for different particle shapes, which worked especially well for clouds with low optical depth. Although this technique may have promising applications, the authors identified that this method only works for top-of-cloud measurements, which would require satellite- or aircraft-based detection.

King et al. ${ }^{31}$ used a large variety of bands that were available on MODIS to retrieve cloud phase. Typically, they determined CTP in a multistep process, starting with thermal infrared bands. If CTP could not be determined using the thermal methods, they used bands within the range of $0.66,1.62$, and $2.13 \mu \mathrm{m}$ to help with the final classification and validated using cloud temperatures from thermal infrared bands.

Martins et al. ${ }^{8}$ tested their cloud scanner prototype that had a scanning spectrometer, thermal scanner, and digital camera fitted with a fisheye lens. They flew an airplane near clouds and measured the scattered sunlight from cloud sides. Their analysis used the 2.10- and 2.25- $\mu \mathrm{m}$ 
bands and confirmed their CTP classifications using the temperature of the clouds provided by the thermal infrared scanner. Clouds that had a temperature between $-40^{\circ} \mathrm{C}$ and $0^{\circ} \mathrm{C}$ were not analyzed because they could have consisted of ice, liquid-water, or mixed-phase particles.

Few researchers have developed their own instrument for CTP detection and validation; however, Jäkel et al. ${ }^{11}$ not only designed a spectrally sensitive instrument, but also used a lidar system to validate their CTP classifications. They defined their phase index as the difference between the $1.55-$ and $1.70-\mu \mathrm{m}$ bands divided by the $1.70-\mu \mathrm{m}$ band. Their instrument was ground-based and operated alongside their dual-polarization lidar. The two instruments pointed in the same direction but with different fields of view (about $1 \mathrm{deg}$ for the spectroradiometer and 0.1 deg for the lidar).

Finally, Thompson et al. ${ }^{10}$ not only reviewed four of the methods discussed above but also proposed a method of spectrum fitting in the 1.4- to $1.8-\mu \mathrm{m}$ range. They used the fit to identify liquid-water content and ice-water content of a cloud. This method improved accuracy in CTP classification relative to some of the methods discussed above. Their remote data were from aircraft flights over ocean water and they used in situ measurements to validate CTP. They recognized the dependence of CTP classification on cloud optical depth and thus did not attempt to classify clouds with an optical depth below 0.25 .

Most of the instruments presented were either satellite based or airborne. These typically downward-looking orientations have the major advantages of covering large areas quickly and operating above the thickest part of the atmosphere where gaseous absorption is the highest. However, airborne and satellite-based remote sensing is costly and downward-viewing measurements of clouds are often contaminated by surface reflections. Furthermore, nearly all methods were declared to be relatively insensitive to viewing geometry; those researchers who noticed a dependence on solar angles stated that the difference was insignificant for CTP retrieval. ${ }^{11}$ However, with the exception of one classification method (which only works for top-of-cloud measurements), ${ }^{33}$ these methods only performed well for clouds with large optical depths. Ground-based spectral radiometers have the disadvantage of thick atmosphere, a static viewing region, and sparse distribution across the globe, but upward-looking instruments, such as the spectrometer used by Pilewskie and Twome ${ }^{23}$ and the spectrometer used by Jäkel et al., ${ }^{11}$ have a simpler background (the sky) and are often cheaper and easier to deploy than air- and spacebased instruments. Therefore, there is an opportunity for ground-based, passive remote sensing radiance measurements.

None of the techniques described so far have used sunlight transmitted through clouds for CTP retrieval. The intent of cloud-side retrievals is often for detailed cloud layer information, and downward looking methods must account for their background, without which CTP differentiation becomes much more difficult, if not impossible. A zenith-pointed instrument would integrate the thermodynamic phase information from the entire vertical column of a cloud, and thus give a general classification instead of detailed spatial information. This is an important step prior to deeper investigations. Furthermore, optical depth is a limiting factor in CTP classification with spectral band ratios, but few, if any, have focused on pushing the boundaries to find the lower limit of optical depth for which a method would still accurately identify CTP. A method that can classify CTP at very low optical depths would be useful since thin clouds are frequent and important for understanding radiative transfer. In this paper, we describe a method that intends to accomplish thermodynamic phase classification based on transmitted sunlight even for clouds with low optical depths.

The remainder of this paper describes simulations and experimental results that identified and demonstrated three spectral bands in the SWIR useful for CTP discrimination. First, we report the use of the MODTRAN (version 5.2.0.0) radiative transfer code to simulate sunlight transmitted through various cloud types. This analysis led us to develop a CTP passive sensing method based on radiance measurements at $1.55,1.64$, and $1.70 \mu \mathrm{m}$. Next, we describe the experimental measurements made with a spectrometer at these three bands, validated with dual-polarization lidar measurements of CTP. Finally, we show that three-dimensional analysis of spectral band ratios provided CTP discrimination for clouds with optical depths as low as $\sim 0.25$ (uncertainties in cloud optical depth for the experimental data and limited ability of the simulations to represent reality prevent us from stating an exact value of the minimum cloud optical depth, but a more detailed discussion is presented in Sec. 3.3). 


\section{Radiative Transfer Simulations}

Simulations were accomplished through analysis of spectral radiance outputs for different cloud types and ground viewing conditions via the MODTRAN radiative transfer code. These simulations are described in more detail in a thesis. ${ }^{34}$ Six standard cloud types were used in the analysis to simulate different droplet distributions at different altitudes. Five of the cloud types, cumulus, altostratus, stratus, nimbostratus, and stratocumulus, had a liquid-water composition, whereas cirrus had an ice composition. The optical depth of each cloud was varied by changing the extinction ratio of the cloud while keeping altitude and geometric thickness at typical values.

The scenario was set as follows: an observer with an altitude at ground level of Bozeman, Montana (1460-m elevation), viewed a cloud at 0-deg azimuth, 45-deg elevation. The sun was behind the observer at 180-deg azimuth, 45-deg elevation. Spectral radiance was then calculated over the 1.40- to $2.10-\mu \mathrm{m}$ band. A distinct difference was seen in the slope of spectral radiance between 1.50 and $1.70 \mu \mathrm{m}$ when comparing ice clouds to liquid-water clouds at the same cloud optical depth. Figure 2 shows this difference for clouds with optical depth of 6 (left) and for clouds with optical depth of 0.1 (right). Although both plots show a distinction in slope between liquid water and ice, the difference is more drastic for optically thick clouds.

A sensitivity analysis was performed to determine the best discrimination bands and bandwidths required to characterize cloud phase. Different algorithms were explored using the relative magnitudes of each sub-band within the 1.50 - to $1.70-\mu \mathrm{m}$ wavelength spectrum. Varying amounts of precipitable water vapor (PWV) caused some issues when discriminating between two channels but using three separate channels within the $1.50-$ to $1.70-\mu \mathrm{m}$ band helped this issue significantly. It was found that the best cloud phase discrimination algorithm involved the use of three spectral bands centered at $1.55,1.64$, and $1.70 \mu \mathrm{m}$ with full-width at half-maximum bandwidths of $150 \mathrm{~nm}$ or less. The normalized difference ratios, $R_{1.70,1.64}, R_{1.55,1.64}$, and $R_{1.55,1.70}$, were then defined by the following equations:

$$
\begin{aligned}
& R_{1.70,1.64}=\frac{L_{1.70}-L_{1.64}}{L_{1.64}}, \\
& R_{1.55,1.64}=\frac{L_{1.55}-L_{1.64}}{L_{1.64}}, \\
& R_{1.55,1.70}=\frac{L_{1.55}-L_{1.70}}{L_{1.70}},
\end{aligned}
$$

where $L_{\text {band }}$ is the detected radiance in that particular spectral band.

Because previous research showed that CTP algorithms worked better for clouds with high optical depth than for clouds with low optical depth, we ran models for clouds with varying optical depths. A three-dimensional plot from these simulations is shown in Fig. 3. The three distinct cases are clear sky (shown as black circles), liquid water (shown as orange lines), and ice (shown as green lines). Each case is plotted multiple times with varying PWV. The most
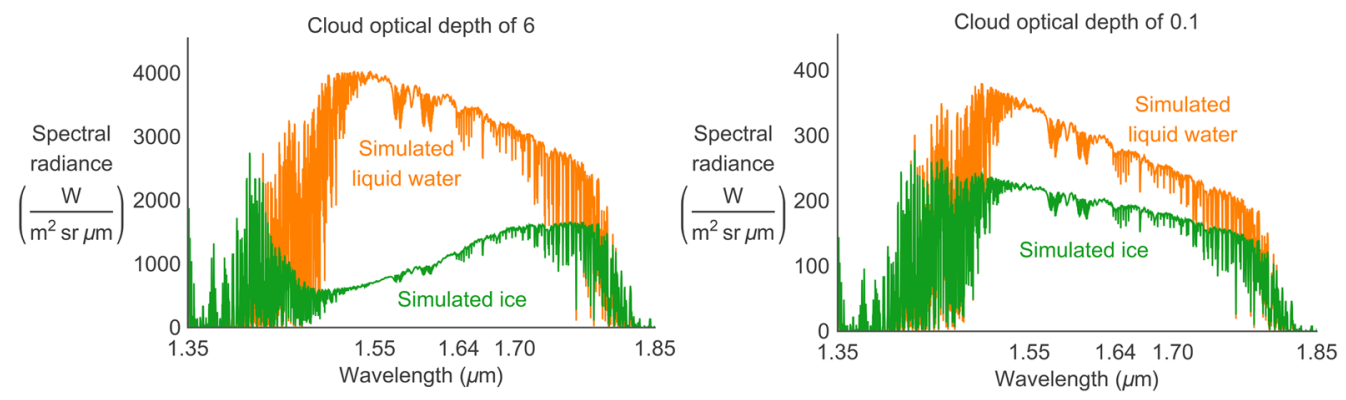

Fig. 2 When the optical depth of the cloud is large, ice clouds (green) show a significant difference in slope from 1.50 to $1.70 \mu \mathrm{m}$ when comparing to water clouds (orange). When the cloud optical depth is low, the slopes become more difficult to distinguish. 


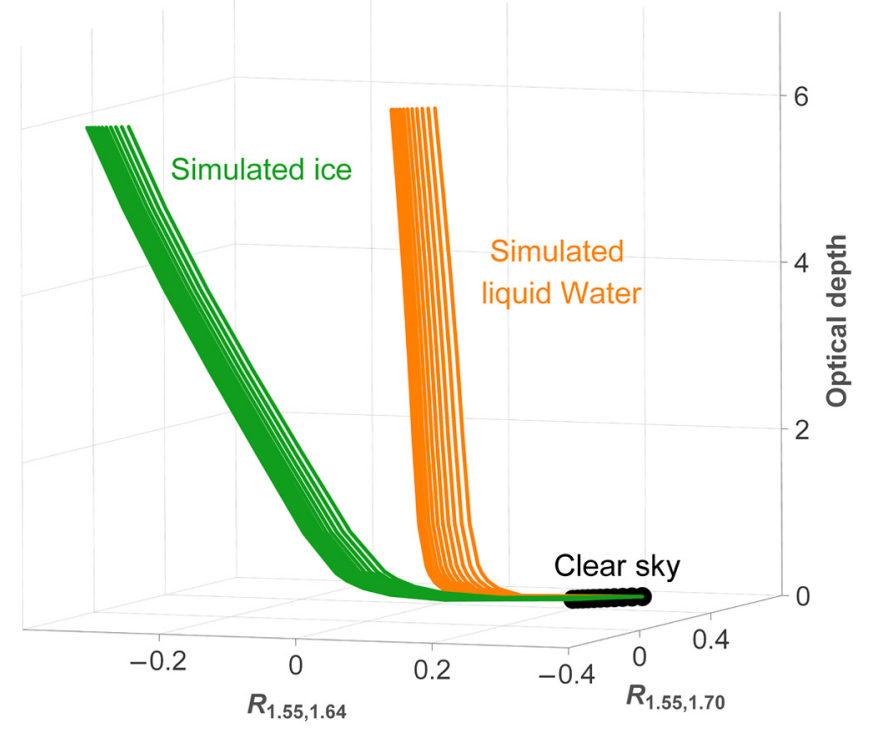

Fig. 3 Three integrated 150-nm-wide bands allow reasonable discrimination of optically thick clouds when evaluated in three dimensions. The curves merge for low cloud optical depth. Various lines represent varying PWV from 0.5 to $1.5 \mathrm{~mm}$. Clear sky is depicted by circles.

noticeable trend is that liquid water and ice are well separated for large cloud optical depths, which means that this method can be used to discriminate between the two. As the cloud optical depth gets smaller, the ability to distinguish between the two becomes impossible. There are two major problems with this scenario: first, cloud optical depth values are typically not available from passive sensors and so we cannot use this space (i.e., optical depth, $R_{1.55,1.64}$, and $R_{1.55,1.70}$ ) to identify a threshold for CTP discrimination; second, this method only works for clouds with large optical depths. The first issue can be solved by swapping the optical depth axis with the third difference ratio $R_{1.70,1.64}$ as indicated in Fig. 4.

Figure 4 does not have as large of a separation as Fig. 3, but each axis represents a readily measurable quantity. In Fig. 4, cloud optical depth is represented figuratively by symbol size (i.e., large pluses or squares represent clouds with large optical depth). As the symbols decrease in size, they approach the bottom right (clear sky). This method of analysis looks promising for clouds with large or even moderately small optical depth (above 0.25 ).

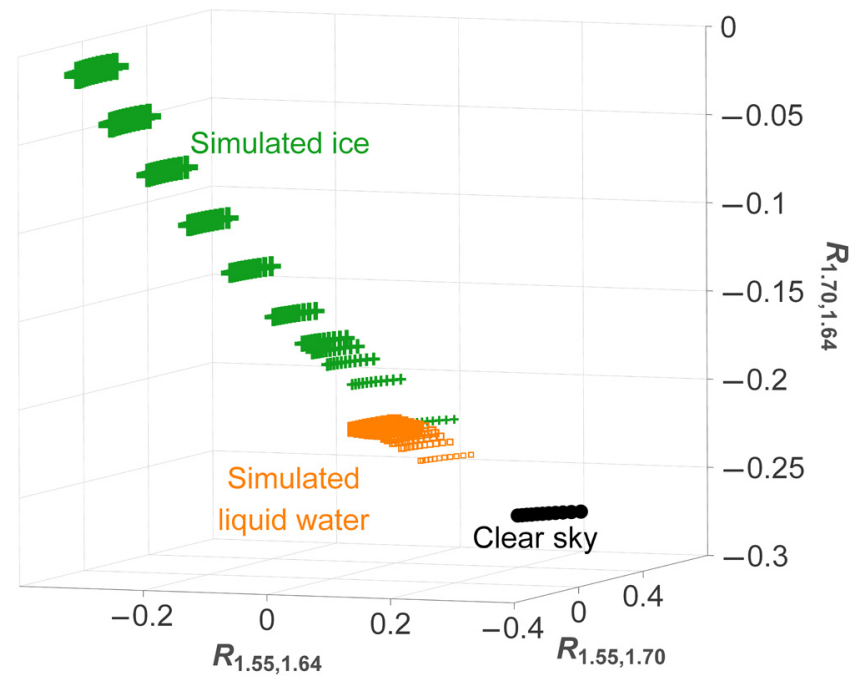

Fig. 4 Plotting the three normalized difference ratios shows good discrimination between ice and liquid-water clouds. Symbol sizes (i.e., pluses and squares) represent optical depth, and as the optical depth decreases, the two cloud types become indistinguishable. 


\section{Experimental Validation}

To test the simulated results, we collected downwelling spectral radiance data using a spectrometer (Analytical Spectral Devices FieldSpec Pro FSP 350-2500P with sensitivity from 0.35 to $2.50 \mu \mathrm{m})$. From the radiance spectra, we binned data to create three spectral channels centered at $1.55,1.64$, and $1.70 \mu \mathrm{m}$ to calculate experimentally determined spectral ratios from Eqs. (1) to (3). We also operated a dual-polarization lidar at the same time to provide ground-truth CTP measurements since the polarization lidar method is so well established for CTP classification. Data were collected during cloudy conditions on January 21 and 23, March 21 and 28, and April 6 and 7, 2016. The timestamps from the spectrometer and from the dual-polarization lidar were compared and overlapping sessions were flagged for analysis. Data from the dual-polarization lidar were manually searched for timestamps that contained liquid-water clouds and timestamps that contained ice clouds. Response-calibrated radiance spectra for liquid-water and ice clouds were processed and analyzed.

\subsection{Spectrometer Calibration}

Although the spectrometer had three separate detectors to achieve a very broad spectral coverage, only the middle detector, with sensitivity between 0.966 and $1.755 \mu \mathrm{m}$, was used due to software limitations. The full-width at half-maximum bandwidths of all channels was $150 \mathrm{~nm}$, except for the $1.70-\mu \mathrm{m}$ channel, whose bandwidth was truncated at the $1.755-\mu \mathrm{m}$ middle-detector cutoff. Figure 5 represents the spectral bands that were used in both the simulations and experiments. A full radiometric calibration was not necessary since the analysis was done on normalized difference ratios and not radiance values. Therefore, the spectrometer was only partially calibrated, as described next.

The middle detector in the spectrometer was response calibrated using the sun, a Spectralon diffuse reflectance target, with Lambertian reflectance of $\sim 95 \%$ in the 1.40 - to $1.80-\mu \mathrm{m}$ range, and a model of solar transmittance with the MODTRAN radiative transfer code. To calibrate the spectrometer, the processes used the sun (on a clear day) as the source and the reflectance target to create a uniform target. The target was placed so its surface was nominally normal to the sun, and the fiber from the spectrometer was mounted so it was viewing near the normal of the reflectance panel. It then recorded the solar spectral radiance as it was transmitted through the atmosphere and reflected from the panel. Then the MODTRAN radiative transfer code was used to model the atmospheric conditions to characterize the signal seen by the spectrometer. Digital number on the spectrometer could then be matched to an estimated radiance value calculated from the MODTRAN radiative transfer code. The spectral shape in the middle detector appeared to not change as a function of solar irradiance, gain, or offset. We did notice that gain and offset values would affect the relationship between the three channels, which is why the middle channel was the only one used.

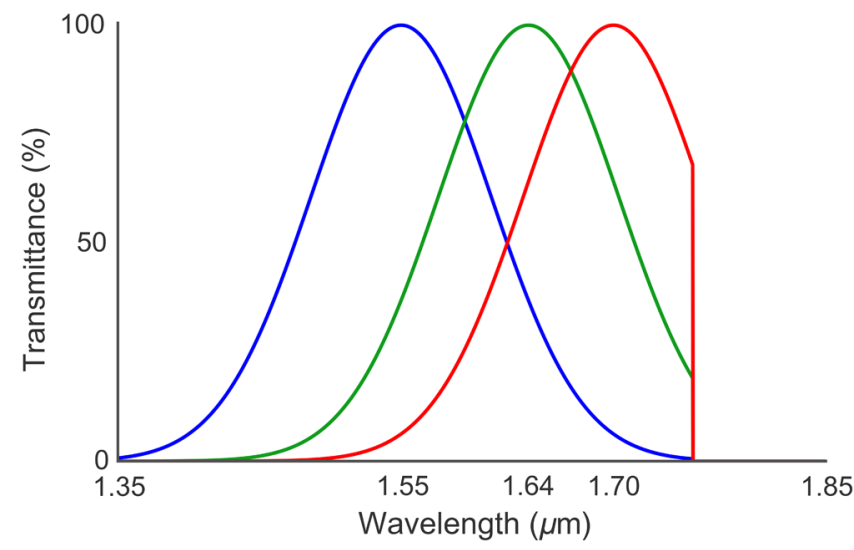

Fig. 5 The three spectral bands used for CTP classification were centered at 1.55, 1.64, and $1.70 \mu \mathrm{m}$ with $150 \mathrm{~nm}$ bandwidths. The spectrometer was only sensitive out to $1.755 \mu \mathrm{m}$, which is why there is a sharp cutoff at this wavelength. 


\subsection{Experiment}

The experimental setup was identical for each data collection session. The input fiber for the spectrometer was mounted $\sim 5 \mathrm{~cm}$ from the receiver of the dual-polarization lidar. The probe was housed in a lens tube assembly to reduce the spectrometer's field of view. Then the lidar would run and classify clouds. Clouds of mixed phase or multiple cloud layers were ignored and only instances of pure ice or pure liquid water were considered. The spectrometer would run in tandem and simply store the full spectrum every few seconds.

After data collection was complete, we interpreted CTP from the dual-polarization lidar signal and flagged the corresponding spectrum either as liquid water or ice, using the standard cross-polarization ratio method described by Sassen $^{21}$ and implemented in the manner discussed by Seldomridge et al. ${ }^{7,22}$ Data from the flagged sessions were calibrated, integrated across the three spectral bands (from Fig. 5), and the three normalized difference ratios were calculated from Eqs. (1)-(3).

\subsection{Analysis}

The three ratios from experimental data are plotted in Fig. 6 to show that liquid-water clouds (blue circles) can be distinguished from ice clouds (red triangles). Simulated liquid-water and ice ratios are included for comparison. Overall, the liquid water and ice are well separated and the experimental results generally match the simulations.

The experimental ice cloud data dominate the upper portion of the plot and, for the most part, closely match the simulated data. The experimental ice points toward the far right portion of this figure are in the general vicinity of, but not exactly coincident with, the simulated results; this is likely caused by certain cloud properties that were not considered in the simulations, such as particle shape, size, and orientation. The MODTRAN code assumes spherical ice particles with radii between 10 and $200 \mu \mathrm{m}$ (Ref. 35) and does not consider the broad variety of cloud microphysical properties that could influence the measurements. It is also possible that artifacts from the experimental setup, such as a fast-moving cloud with varying properties averaged over the spectrometer's exposure time, contributed to the difference between simulated and measured values. Nevertheless, the important point is that the measured ice cloud data are well separated from the measured liquid cloud data.

The experimental data from liquid-water clouds are very consistent with the simulated data for low cloud optical depths. In Fig. 6, the experimental data overlap for the available simulated

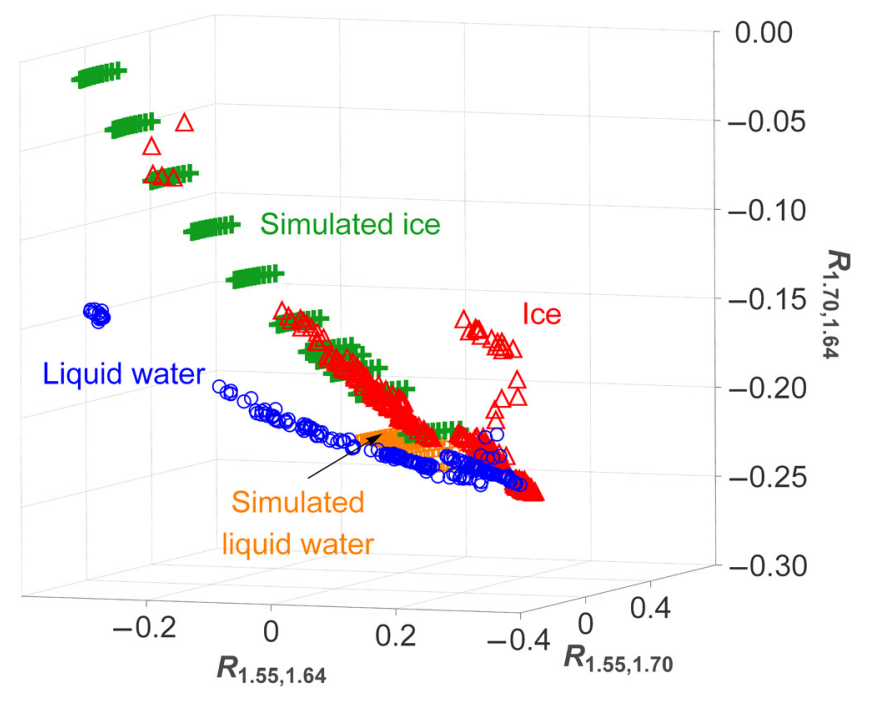

Fig. 6 The data collected from the spectrometer are plotted for liquid-water (blue circles) and ice (red triangles) clouds in three dimensions and the simulated data are added in for comparison. Although the two cloud types converge when cloud optical depths are low, they are otherwise well separated. 
data (i.e., for optical depth values between 0.1 and 6). If simulated data for liquid-water clouds were extrapolated into higher and lower optical depths, they would also overlap with experimental data, suggesting that the clouds from the experiments had a very wide range of optical depths. The overall pattern of liquid-water clouds from the experiments matches the nominal pattern from the simulations in that it extends in the expected direction for larger and smaller optical depths. As shown in Fig. 4, as the optical depth became smaller, the values approached the bottom right corner of the figure.

The fact that low cloud optical depths hinder CTP classification is not surprising. Using spectral ratios for classification relies on the different absorption properties of liquid water and ice as opposed to scattering behavior from cloud particles. As a cloud's optical depth decreases, fewer photons have the opportunity to be absorbed and so classifying the thermodynamic phase becomes more difficult. Because cloud optical depth has such a strong impact on the ability to discriminate clouds, identifying a cutoff value is important. The bottom right part of Fig. 6 represents values of low cloud optical depth and so a fair amount of mixing between liquid-water and ice measurements is apparent. We attempt to interpret the cut-off value of optical depth for which liquid-water and ice clouds cannot be distinguished. By interpolating PWV values at low values of optical depth, it appears as though a liquid-water cloud of optical depth near 0.5 and an ice cloud of $<0.1$ occupy the same region and so represent threshold values above which this method appears to be effective.

In general, the experimental results (gathered over the course of six days in January, March, and April) matched the simulated data. Although clouds became indistinguishable when their optical depth was very low, they were well separated otherwise. When the cloud optical depth was above some threshold (theoretically above $\sim 0.25$, and experimentally above 0.5 for liquid water and 0.1 for ice), the two thermodynamic phases became distinguishable. Most studies have found that CTP classification breaks down at low optical depths ${ }^{5,10}$ (typically below at least 1 for methods using spectral band ratios), limiting the usability of spectral analysis for discrimination. Even though our method also breaks down at very low optical depths, it only misclassifies $2.9 \%$ of data when setting a threshold plane where values above the plane correspond to ice clouds and values below to liquid-water clouds. The plane, defined by

$$
R_{1.70,1.64}=A R_{1.55,1.64}+B R_{1.55,1.70}+C
$$

where $A=0.0272, B=-0.1846$, and $C=-0.1561$, was found by iteratively changing the coefficients until the lowest number of incorrect classifications was found. Such a method of determining a threshold may be subject to overfitting for this specific data set but is reasonable for identifying an initial threshold plane.

A classification error of $2.9 \%$ is good, considering the limits in optical depth. Had our method only considered clouds with larger optical depths, similar to the procedures by other researchers,${ }^{10,19}$ then our classification accuracy would have been higher, because there is obvious mixing of CTP in the region of Fig. 6 for low optical depths. The region in the lower right corner contains clouds with low optical depths, and then both liquid-water and ice cloud measurements with higher optical depths branch toward the upper left. Furthermore, our data set contains a variety of cloud types due to the fact that data collection took place on six different days in winter and spring. This appears to be reflected in the way the experimental data spans a large region of Fig. 6.

As implemented in our upward-viewing experiments, this method benefits from a much simpler background relative to that seen by downward-viewing sensors. We also note that our experiments were made at the zenith for convenience with a zenith-pointed lidar, but this method could be implemented with a sensor pointing at potentially any angle.

An important element of this method is analyzing the data in three-dimensional parameter space, which allows for a threshold plane to classify CTP instead of a single value, which is common in other studies. The plane has three coefficients that allow for fine tuning of the threshold and can better account for optical depth dependence. The use of a plane in this threeparameter space allows for details to be accounted for so that the unique shapes from analysis of spectral band ratios can be separated with finer control. 


\section{Conclusion}

In this paper, we presented a method of determining cloud thermodynamic phase based on the relationship between three normalized difference ratios in the SWIR. Unlike most other studies, we measured solar radiation transmitted through clouds, instead of reflected, and performed our analysis by determining a threshold plane in three-parameter space instead of a single value. The cloud optical depth was a limiting factor in this study, as well as most of those discussed in Sec. 1. Below a cloud optical depth value of $\sim 0.25$, the CTP becomes difficult to distinguish; nonetheless, discrimination based on a best-fit plane misclassified $<3 \%$ of the data using coefficients $A=0.0272, B=-0.1846$, and $C=-0.1561$ in Eq. (4).

Nature often produces cloud conditions that are far more complex than single liquid-water or ice clouds, such as multilayered clouds or mixed-phase clouds, which are even more difficult to classify and distinguish. With additional ground-truth data, the method introduced here could be extended to distinguish mixed-phase clouds, in addition to liquid and ice, similar to what has been done with other methods based on spectral radiance. ${ }^{8,10,11,29-33}$ These methods often relied on setting two thresholds, one for liquid water and one for ice, and classifying mixed-phase clouds in between these two thresholds. Although Thompson et al. ${ }^{10}$ were able to make impressive strides in mixed-phase cloud classifications, their method used a more complicated spectral fitting procedure as compared to our three spectral ratios. The purpose of this study was to establish a starting point for future analysis and instrumentation, focused on cases of pure liquid and ice clouds.

The use of spectral bands in itself is not enough to classify CTP for every cloud in the sky, due to the limitations with optically thin clouds. Additional capabilities are necessary for a passive instrument to detect optically thin clouds. Recently, work in polarimetry has shown some promising results for classifying CTP in the visible and near-infrared spectra for optically thin clouds. $^{36,37}$ A combination of radiance ratios and polarimetry has been realized in an SWIR polarimeter based on the three spectral bands $(1.55,1.64$, and $1.70 \mu \mathrm{m})$ and is currently being tested as a relatively inexpensive passive polarimeter for CTP classification. Such instruments may classify liquid-phase or ice-phase clouds with greater accuracy.

Identifying the thermodynamic phase of a cloud is important for climate and weather studies as well as optical communications with space-based instruments. The work presented in this paper lays the groundwork for a new passive instrument that could be cost-effectively produced so that multiple CTP classifiers can be deployed around the world to improve our general understanding of clouds, our abilities to communicate with space-based instruments, and our understanding of the Earth's climate.

\section{Acknowledgments}

This work was funded by NASA EPSCoR (Grant No. NNX14AN40A) and by the Air Force Office of Scientific Research under Agreement No. FA9550-14-1-0140. The U.S. Government is authorized to reproduce and distribute reprints for Governmental purposes notwithstanding any copyright notation thereon. The views and conclusions contained herein are those of the authors and should not be interpreted as necessarily representing the official policies or endorsements, either expressed or implied, of the Air Force Research Laboratory or the U.S. Government. Portions of this work were published in "Cloud thermodynamic phase detection with a 3-channel shortwave infrared polarimeter," Proc. SPIE 10655, 106550O, 14 May $2018 .{ }^{38}$

\section{References}

1. W. J. Wiscombe, “An absorbing mystery," Nature 376, 466-467 (1995).

2. V. Ramanathan et al., "Warm pool heat budget and shortwave cloud forcing: a missing physics?" Science 267(5197), 499-503 (1995).

3. J. R. Key and J. M. Intrieri, "Cloud particle phase determination with the AVHRR," J. Appl. Meteorol. 39, 1797-1804 (2000).

4. G. Flato et al., "2013: evaluation of climate models," Tech. Rep., Intergovernmental Panel on Climate Change, Cambridge, United Kingdom and New York (2013). 
Tauc et al.: Simulations and experimental results of cloud thermodynamic...

5. W. H. Knap, P. Stammes, and R. B. A. Koelemeijer, "Cloud thermodynamic-phase determination from near-infrared spectra of reflected sunlight," J. Atmos. Sci. 59, 83-96 (2002).

6. W. B. Rossow and R. A. Schiffer, "Advances in understandig clouds from ISCCP," Bull. Am. Meteorol. Soc. 80(11), 2261-2287 (1999).

7. N. L. Seldomridge, J. A. Shaw, and K. S. Repasky, "Dual-polarization lidar using a liquid crystal variable retarder," Opt. Eng. 45(10), 106202 (2006).

8. J. V. Martins et al., "Remote sensing the vertical profile of cloud droplet effective radius, thermodynamic phase, and temperature," Atmos. Chem. Phys. 11(18), 9485-9501 (2011).

9. M. D. King et al., "Cloud retrieval algorithms for MODIS: optical thickness, effective particle radius, and thermodynamic phase," Tech. Rep. 5, NASA Goddard Space Flight Center (1997).

10. D. R. Thompson et al., "Measuring cloud thermodynamic phase with shortwave infrared imaging spectroscopy," J. Geophys. Res. Atmos. 121(15), 9174-9190 (2016).

11. E. Jäkel, J. Walter, and M. Wendisch, "Thermodynamic phase retrieval of convective clouds: impact of sensor viewing geometry and vertical distribution of cloud properties," Atmos. Meas. Tech. 6(3), 539-547 (2013).

12. Y.-X. Hu et al., "Identification of cloud phase from PICASSO-CENA lidar depolarization: a multiple scattering sensitivity study," J. Quant. Spectrosc. Radiat. Transfer 70(4-6), 569-579 (2001).

13. J. H. Churnside and K. Shaik, "Atmospheric propagation issues relevant to optical communications," Tech. Rep., NASA Jet Propulsion Laboratory, Pasadena, California (1989).

14. P. W. Nugent, J. A. Shaw, and S. Piazzolla, "Infrared cloud imager development for atmospheric optical communication characterization, and measurements at the JPL table mountain facility," Tech. Rep. 42-192, Electrical and Computer Engineering Department, Montana State University, Bozeman, Montana (2013).

15. P. W. Nugent, J. A. Shaw, and S. Piazzolla, "Infrared cloud imaging in support of Earthspace optical communication," Opt. Express 17, 7862-7872 (2009).

16. B. A. Baum et al., "Remote sensing of cloud properties using MODIS airborne simulator imagery during SUCCESS: 1. Data and models," J. Geophys. Res. Atmos. 105, 1176711780 (2000).

17. G. L. Stephens et al., "The relevance of the microphysical and radiative properties of cirrus clouds to climate and climatic feedback," J. Atmos. Sci. 47, 1742-1754 (1990).

18. V. Ramanathan et al., "Cloud-radiative forcing and climate: results from the Earth radiation budget experiment," Science 243(4887), 57-63 (1989).

19. J. Acarreta, P. Stammes, and W. Knap, "First retrieval of cloud phase from SCIAMACHY spectra around $1.6 \mu \mathrm{m}$," Atmos. Res. 72, 89-105 (2004).

20. R. M. Schotland, K. Sassen, and R. Stone, "Observations by lidar of linear depolarization ratios for hydrometeors," J. Appl. Meteorol. 10(5), 1011-1017 (1971).

21. K. Sassen, "The polarization lidar technique for cloud research: a review and current assessment," Bull. Am. Meteorol. Soc. 72, 1848-1866 (1991).

22. N. L. Seldomridge, "Dual-polarization cloud lidar design and characterization," Master's thesis, Montana State University (2005).

23. P. Pilewskie and S. Twomey, "Discrimination of ice from water in clouds by optical remote sensing," Atmos. Res. 21(2), 113-122 (1987).

24. B. Holben et al., "AERONET-A federated instrument network and data archive for aerosol characterization," Remote Sens. Environ. 66(1), 1-16 (1998).

25. D. M. Gates and C. C. Shaw, "Infrared transmission of clouds," J. Opt. Soc. Am. 50, 876-882 (1960).

26. S. G. Warren, "Optical constants of ice from the ultraviolet to the microwave," Appl. Opt. 23, 1206-1225 (1984).

27. S. G. Warren and R. E. Brandt, "Optical constants of ice from the ultraviolet to the microwave: a revised compilation,” J. Geophys. Res. 113, D14220 (2008).

28. L. Kou, D. Labrie, and P. Chylek, "Refractive indices of water and ice in the $0.65-$ to $2.5-\mu \mathrm{m}$ spectral range," Appl. Opt. 32, 3531-3540 (1993). 
29. B. A. Baum et al., "Remote sensing of cloud properties using MODIS airborne simulator imagery during SUCCESS: 2. Cloud thermodynamic phase," J. Geophys. Res. Atmos. 105, 11781-11792 (2000).

30. P. Chylek and C. Borel, "Mixed phase cloud water/ice structure from high spatial resolution satellite data," Geophys. Res. Lett. 31(14), L14104 (2004).

31. M. D. King et al., "Remote sensing of liquid water and ice cloud optical thickness and effective radius in the Arctic: application of airborne multispectral MAS data," J. Atmos. Oceanic Technol. 21, 857-875 (2004).

32. P. Chylek et al., "Comparison of near-infrared and thermal infrared cloud phase detections," J. Geophys. Res. 111, D20203 (2006).

33. A. Ehrlich et al., "Cloud phase identification of Arctic boundary-layer clouds from airborne spectral reflection measurements: test of three approaches," Atmos. Chem. Phys. 8, 7493-7505 (2008).

34. D. W. Riesland, "Infrared cloud imaging systems characterization," Master's thesis, Montana State University (2016).

35. D. K. Lynch and S. M. Mazuk, "Wavelength dependence of cirrus optical depth," Tech. Rep. 8570, The Aerospace Corportation (2001).

36. K. Knobelspiesse et al., "Cloud thermodynamic phase detection with polarimetrically sensitive passive sky radiometers," Atmos. Meas. Tech. 8, 1537-1554 (2015).

37. L. M. Eshelman, M. J. Tauc, and J. A. Shaw, "All-sky polarization imaging of cloud thermodynamic phase," Opt. Express 27, 3528-3541 (2019).

38. M. J. Tauc et al., "Cloud thermodynamic phase detection with a 3-channel shortwave infrared polarimeter," Proc. SPIE 10655, 1065500 (2018).

Martin Jan Tauc received his BS degree in physics and mathematics from the University of Oregon and his MS degree in electrical engineering from Montana State University. He is currently a PhD candidate in electrical engineering at Montana State University with a focus on optics and photonics. His current research is on passive polarimetric measurements of the atmosphere.

David W. Riesland received his BS degree in electrical engineering and his MS degree in optics and photonics, both from Montana State University. His current research is in infrared and visible projector technology for the U.S. Army Futures Command, Combat Capability Development Center, Aviation and Missile Center in Redstone Arsenal, Alabama.

Laura M. Eshelman received her BA degree in physics from Gustavus Adolphus College in 2013 and her MS degree in electrical engineering from Montana State University in 2015. She completed her PhD in electrical engineering at Montana State University in 2018. Her graduate work focused on studying atmospheric polarization using all-sky imaging systems. She currently works at Polaris Sensor Technologies in Huntsville, Alabama.

Wataru Nakagawa received his BS degree in physics from Stanford University and his MS and $\mathrm{PhD}$ degrees in electrical and computer engineering (applied physics) from the University of California, San Diego. He is currently an associate professor in the Department of Electrical and Computer Engineering at Montana State University, Bozeman, Montana. His research interests include near-field optical effects in photonic structures and interdisciplinary applications of nanostructured optical devices.

Joseph A. Shaw received his BS degree in electrical engineering from the University of Alaska, his MS degree in electrical engineering from the University of Utah, and his MS and $\mathrm{PhD}$ degrees in optical sciences from the University of Arizona. He is the director of the Optical Technology Center and a professor of optics and photonics and electrical engineering at Montana State University, Bozeman. His research is developing optical remote sensing systems. He is a fellow of OSA and SPIE. 